\title{
A Cascaded Algorithm for Image Quality Assessment and Image Denoising Based on CNN for Image Security and Authorization
}

\author{
Jianjun Li, ${ }^{1}$ Jie Yu $\mathbb{D},{ }^{1}$ Lanlan Xu, ${ }^{1}$ Xinying Xue, ${ }^{1}$ Chin-Chen Chang ${ }^{\mathbb{D}},{ }^{2}$ \\ Xiaoyang Mao, ${ }^{3}$ and Junfeng $\mathrm{Hu}^{4}$ \\ ${ }^{1}$ School of Computer Science and Engineering, Hangzhou Dianzi University, China \\ ${ }^{2}$ Department of Information Engineering and Computer Science, Feng Chia University, Taiwan \\ ${ }^{3}$ Department of Computer Science and Media Engineering, University of Yamanashi, Kofu, Japan \\ ${ }^{4}$ Key Lab of Data Link, China Electronics Technology Group Corporation, Xi'an, China \\ Correspondence should be addressed to Chin-Chen Chang; alan3c@gmail.com
}

Received 15 March 2018; Revised 26 June 2018; Accepted 8 July 2018; Published 17 July 2018

Academic Editor: Ilsun You

Copyright (C) 2018 Jianjun Li et al. This is an open access article distributed under the Creative Commons Attribution License, which permits unrestricted use, distribution, and reproduction in any medium, provided the original work is properly cited.

\begin{abstract}
With the rapid development of Internet technology, images on the Internet are used in various aspects of people's lives. The security and authorization of images are strongly dependent on image quality. Some potential problems have also emerged, among which the quality assessment and denoising of images are particularly evident. This paper proposes a novel NR-IQA method based on the dual convolutional neural network structure, which combines saliency detection with the human visual system (HSV), used as a weighting function to reflect the important distortion caused by the local area. The model is trained using gray and color features in the HSV space. It is applied to the parameter selection of an image denoising algorithm. The experiment proves that our proposed method can accurately evaluate image quality in the process of denoising. It provides great help in parameter optimization iteration and improves the performance of the algorithm. Through experiments, we obtain both improved image quality and a reasonable result of subject assessment when the cascaded algorithm is applied in image security and authorization.
\end{abstract}

\section{Introduction}

With the continuous increase in the size of video surveillance networks, current large-scale video surveillance systems contain tens of thousands of cameras and millions of millions of images or pictures. However, they may be interfered and attacked [1,2] during authorization, encryption [3], acquisition, coding, and transmission. As a result, the quality of video has deteriorated, which has affected the development of related work. In terms of artificial intelligence, for example, image content analysis, text detection, and recognition of traffic signs [4] require high image quality to ensure the accuracy and reliability of detection and recognition.

Today's systems of security and authorization are very large and there are millions of images and videos produced by the Internet every day. Under such circumstances, it is unrealistic to employ a large number of people to subjectively evaluate the quality of each monitored image and video.
Therefore, how to objectively evaluate such quality to meet the needs of monitoring and authorization has become a new direction of research in the field of security and authorization.

Image quality assessment is divided into subjective and objective evaluation methods. The subjective evaluation method [5-7] mainly evaluates image quality through human viewing and is also suitable for the evaluation of video image quality. However, this method is influenced by both subjective human factors and objective factors arising from the observation environment. Therefore, the stability of the evaluation results is poor. An objective evaluation method is based on a mathematical model, which has the advantages of simple operation, fast calculation speed, and the ability to be embedded in the system. Objective evaluation methods of image quality are divided into three types: no parameter, semiparameter, and parameter. This article is based on the actual situation of the application, using the parameter-free algorithm for image distortion. 

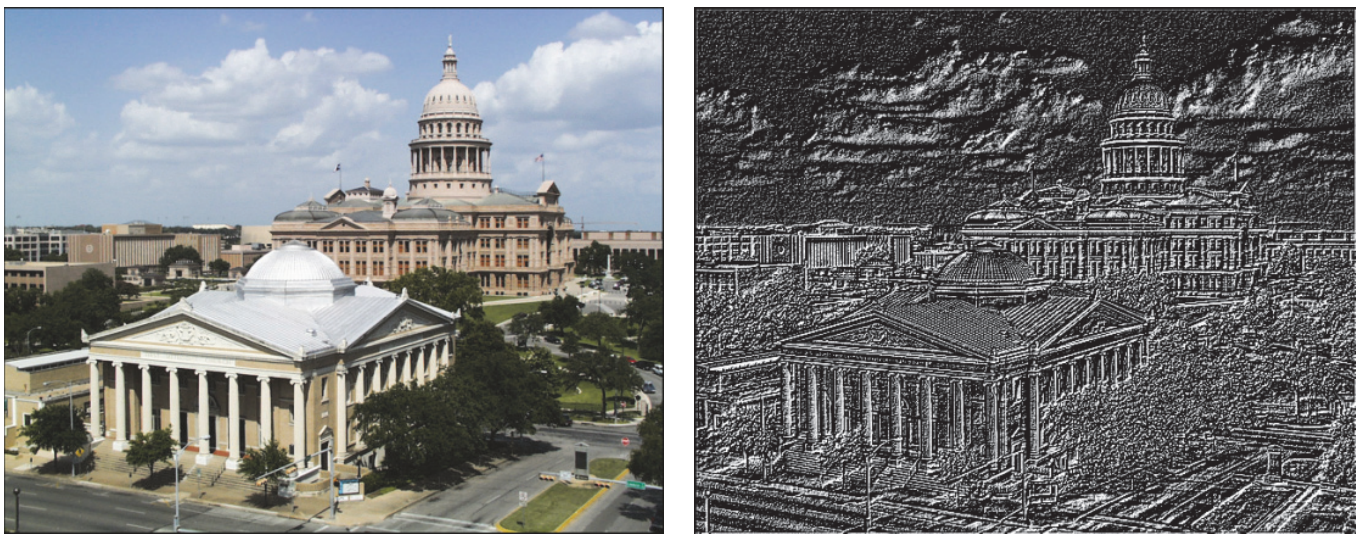

FIGURE 1: Example of image local normalization.

A no-reference image quality assessment (NR-IQA) method can not only evaluate image processing algorithms but also optimize the image system $[8,9]$. At present, remarkable results have been achieved. Therefore, this paper proposes a parameter selection for a denoising algorithm based on the evaluation of no-reference image quality [10] and applies the no-reference image quality evaluation method to the image denoising algorithm [11-13], making it possible to adaptively select the optimal parameters. We embed it in the ROF model to experiment [14] in the search for the optimal parameters, while reducing the number of iterations of the algorithm. This achieved the best denoising effect.

\section{Related Work}

2.1. Image Preprocessing. Image preprocessing is a very important part of the image system. It can provide reliable data for subsequent image processing, thereby improving detection and recognition accuracy. There are many methods, such as image denoising and image enhancement, which can eliminate interference information and purposefully enhance useful information respectively. However, there are very few methods of image preprocessing in the field of image quality evaluation, because most preprocessing operations will enhance or reduce the distortion information in the image. The existing no-reference image quality evaluation methods, such as those discussed in the literature $[15,16]$, use local contrast normalization as a preprocessing operation. As Ruderma described in [17], image local normalization has the effect of image decorrelation. Given an image, the grayscale image is obtained and each pixel in the image is subtracted from its local mean and divided by its local variance, as shown in Figure 1. Assuming that $I(i, j)$ is the pixel value at position $(i, j)$ in the image, the local comparison operation is normalized:

$$
\widehat{\mathrm{I}}(i, \mathrm{j})=\frac{I(\mathrm{i}, \mathrm{j})-\mu(i, \mathrm{j})}{\sigma(\mathrm{i}, \mathrm{j})+\mathrm{C}}
$$

where $i \in 1,2, \ldots, M, j \in 1,2, \ldots, N, M$ and $N$ are the height and width of the image, and $C$ is a positive constant to avoid having the denominator be zero.

$$
\begin{aligned}
& \mu(\mathrm{i}, \mathrm{j})=\sum_{k=-K}^{K} \sum_{l=-L}^{L} \omega_{k . l} I_{k . l}(i, j) \\
& \sigma(\mathrm{i}, \mathrm{j})=\sqrt{\sum_{k=-K}^{K} \sum_{l=-L}^{L} \omega_{k . l}\left(I_{k . l}(i, j)-\mu(i, j)\right)^{2}}
\end{aligned}
$$

where $w=\left\{w_{k, l} \mid k=-K, \ldots, K, l=-L, \ldots, L\right\}$ is a $2-$ dimensional symmetric Gaussian weighting function and $K$ and $L$ are the normalized window sizes. The literature [15] shows that when calculating the local mean and variance, the performance of the algorithm is relatively stable with the change of window scale. However, when the window scale becomes very large, the performance of the algorithm will begin to decrease because the calculated mean and variance are no longer local information.

2.2. Visual Saliency. In the method proposed by Le Kang, the average value of all local quality scores for the test image is taken as the quality value for the entire image. However, such methods do not take into account human visual perception of the image, because the content of the local image block and its position in the entire image will affect the viewer's visual perception. For example, people are less sensitive to distortion in flat areas of an image (such as blue sky) than in complex textured areas (such as edges). Each block in the image has a different effect on human-perceived image quality. We therefore use the saliency of image blocks in the following study as a weight to represent the degree of human visual perception. Figure 2 shows how to predict a saliency map.

\section{Method}

3.1. Input. Previous NR-IQA methods based on deep learning, such as $[16,18]$, consider only the information of grayscale images and ignore the distortion information contained in the color components of the image. Yet, as can be seen in Figure 3, distortion has the most significant effect on the hue component. Therefore, we use HSV as the color space and use the image hue component and grayscale information 


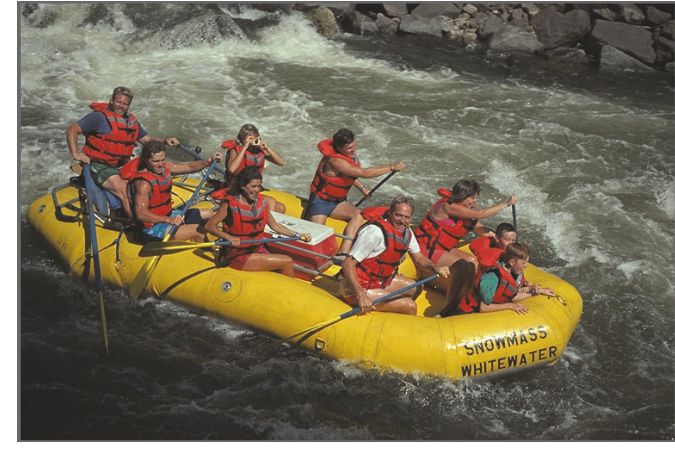

(a)

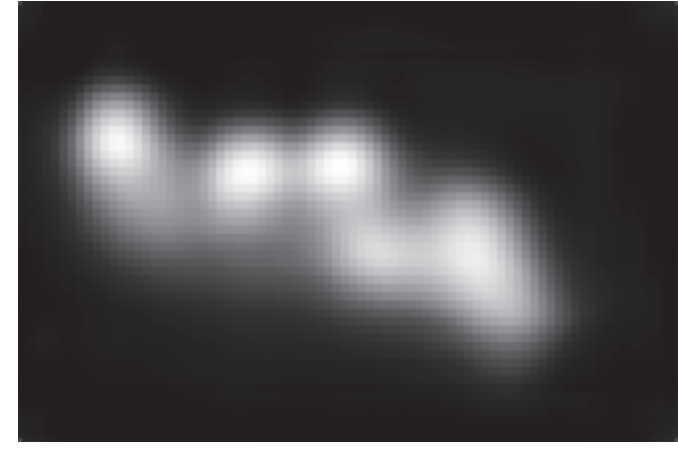

(b)

FIGURE 2: Schematic detection results: (a) original image and (b) saliency map.

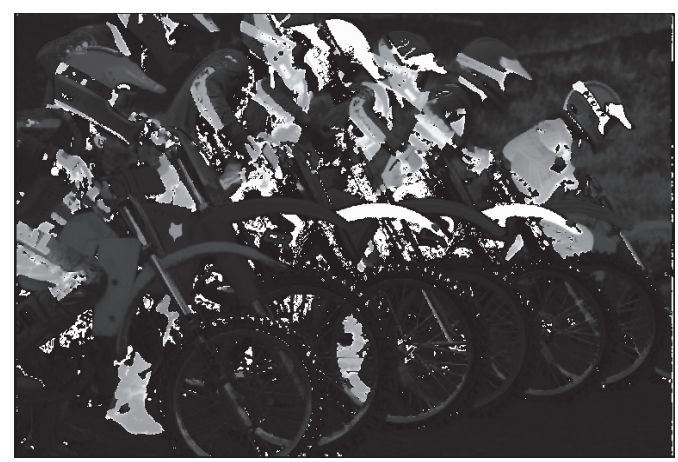

(a)

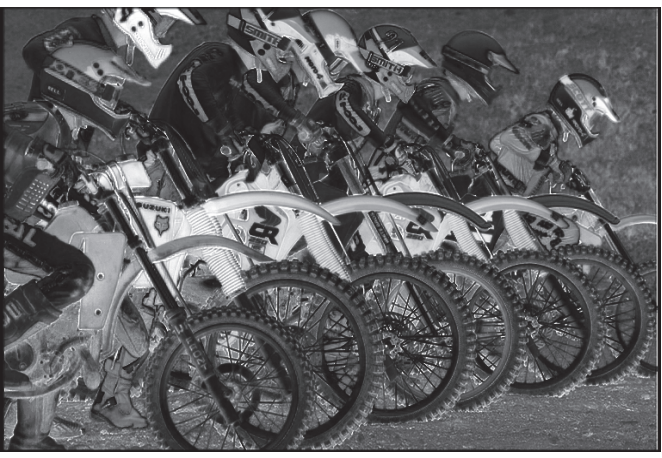

(c)

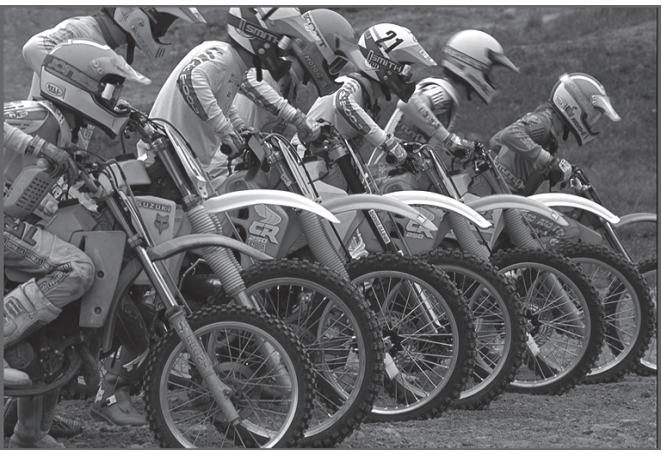

(e)

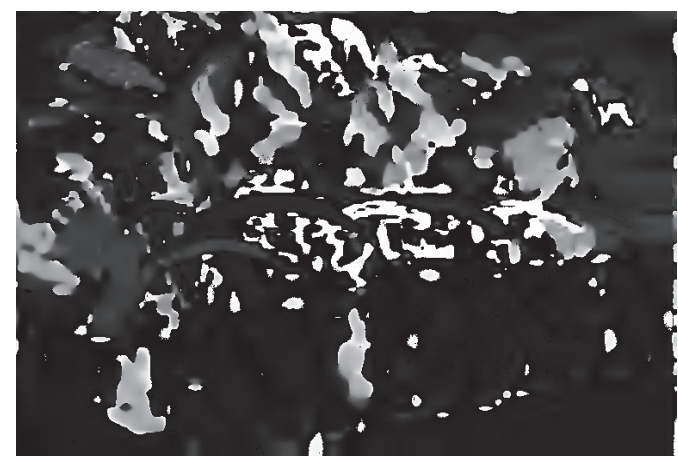

(b)

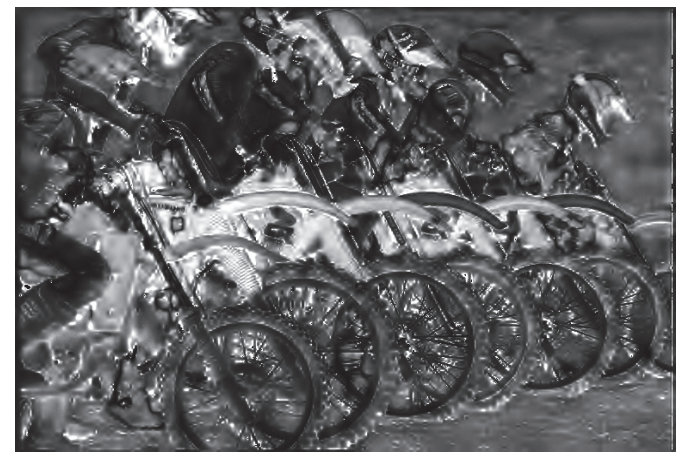

(d)

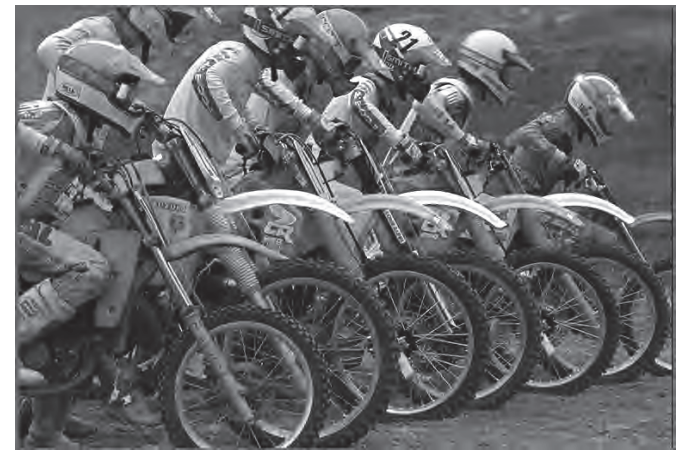

(f)

FIgURE 3: The representation of an image in the HSV color space. The first column contains the three components of the reference image in the HSV color space. The second column is the three components of the JPEG2000 distorted image in the HSV color space. (a) and (b) represent a hue component; (c) and (d) represent a saturation component; (e) and (f) represent a value component. 


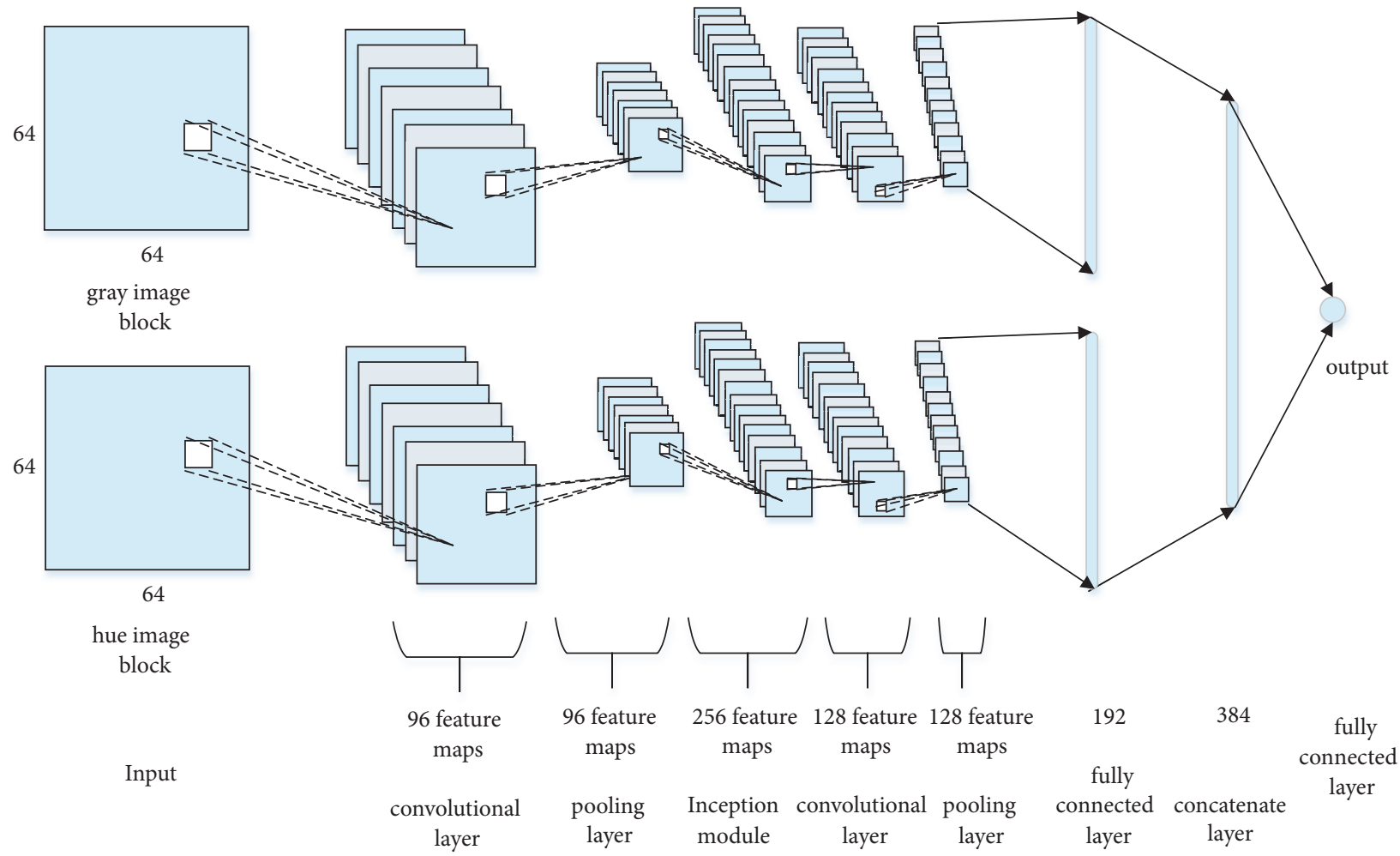

FIGURE 4: CNN model architecture.

as the input for the CNN model to extract features related to image distortion.

3.2. CNN. In this section, we will introduce the proposed CNN model in detail. Figure 4 shows the network structure of the model. It consists of two parts. Apart from the different inputs, the other structures, including the number and size of the convolution kernels, are the same. One of the inputs is the gray scale image block and the other input is the hue image block. The size of the block is $64 \times 64$. For the network, the first layer is a convolutional layer with 96 convolutional kernels with size of $5 \times 5$ and stride of 2 pixels for each. It produces 96 feature maps with size of $30 \times 30$ for each. A max pooling layer follows the convolutional layer to reduce each feature map to size of $15 \times 15$. After the pooling layer, there is an inception module that produces 256 feature maps with size of $15 \times 15$ for each. The next layer is a convolutional layer with 128 convolutional kernels with size of $3 \times 3$ and a padding of 1 pixels. It produces 128 feature maps with size of $15 \times 15$ for each. Following the convolutional layer is a max pooling layer that samples feature maps to a size of $7 \times 7$. The sixth layer is a fully connected layer of 192 nodes. The next layer is a concatenate layer that connects two feature vectors with dimension of 192 for each layer. It has two components. The last layer is also a fully connected layer that gives the quality score of the image block. Except for the last fully connected layer, each layer is followed by a ReLUs (Rectified Linear Units) activation layer.
In addition, to prevent overfitting when training the $\mathrm{CNN}$ model, we apply dropout regularization with a ratio of 0.5 before the last fully connected layer. Because image quality assessment is a quantitative problem, the CNN needs continuous variable prediction. Thus, the Euclidean distance loss function is chosen as the learning loss function:

$$
\mathrm{E}=\frac{1}{2 N} \sum_{n=1}^{N}\left\|\widehat{y}_{n}-y_{n}\right\|_{2}^{2}
$$

where $\widehat{y}_{n}$ is the image block quality score predicted by CNN, $y_{n}$ is the image block quality score, and $N$ is the number of blocks.

3.3. Image Quality Assessment. Through the proposed CNN model, the local image quality evaluation can be obtained. An RGB image is given, converted into a grayscale image and a hue image in the HSV color space, and then the grayscale and hue images are sampled separately. The sampled image block is then subjected to a preprocessing operation, i.e., the local contrast normalization described in (3). Figure 5 is a preprocessed gray image. Similarly, the hue image is subjected to the same sampling operation and preprocessing to obtain a corresponding hue image block. Finally, the prepared gray image block and the hue image block are inputted in pairs into the CNN model, and a series of convolution and pooling processes are performed to output the image block quality value, i.e., the local image quality evaluation. 


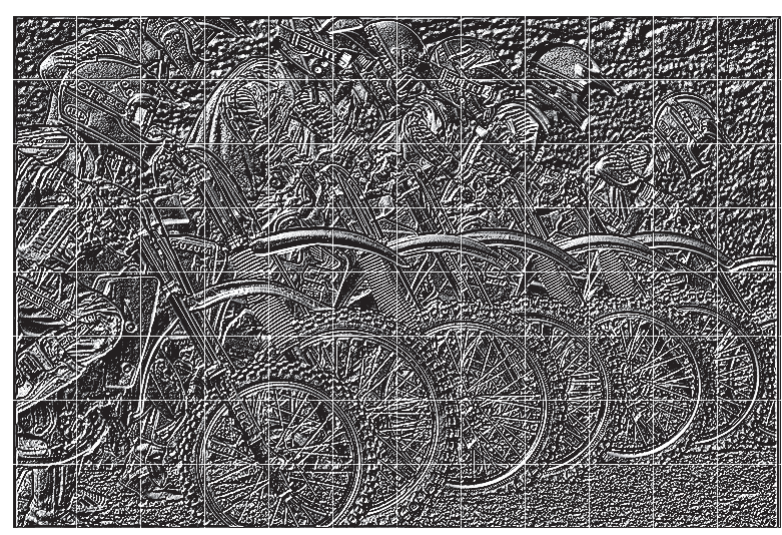

FIgURE 5: Preprocessed grayscale image blocks.

For a test image $\mathrm{G}$, we obtain the corresponding saliency map $S$ through the saliency detection model. In order to obtain the image block weight corresponding to the CNN model, we adopt a nonoverlapping sampling strategy for the saliency map S. The weight of the image block is expressed as

$$
\begin{aligned}
\mathrm{c}_{k} & =\sum_{m=-H}^{H} \sum_{n=-H}^{H} p_{s, k}(i, j) \\
\mathrm{w}_{k} & =\frac{c_{k}}{\sum_{l=1}^{l} c_{k}}
\end{aligned}
$$

For test image $G=\left[P_{g, 1}, P_{g, 2}, \ldots, P_{g, l}\right]$ and saliency map $S=$ $\left[P_{s, 1}, P_{s, 2}, \ldots, P_{s, l}\right], l$ is the number of blocks. There is a one-toone correspondence between $P_{g}$ and $P_{s} \cdot p_{s, k}(i, j)$ is the pixel value of the position $(i, j)$ in the significant image block $P_{s, k}$, and $c_{k}$ is the sum of the coefficients of the image block $P_{s, k}$. Finally, we calculate the global quality score of test image G:

$$
\mathrm{Q}=\sum_{k=1}^{l} w_{k} q_{k}
$$

The algorithm structure is shown in Figure 6.

3.4. Image Denoising. Nowadays, many algorithms [19-21] have achieved very good denoising effects. For example, Rudin et al. [21] proposed a classic total variation (TV) denoising algorithm in 1992, namely, the ROF model. However, an iterative denoising algorithm is always computationally intensive since it requires multiple rounds of image quality assessment to find the best parameters. To solve this problem, we propose a parameter selection framework which reduces the number of iterations of the algorithm while finding the optimal parameters, in order to save execution time.

3.4.1. No-Reference Image Quality Assessment. The assessment method for image quality will be applied to an image processing algorithm to achieve optimal parameter selection. Based on the success of the CNN model for image quality assessment in Section 3.3, we propose a simple CNN model for evaluating the quality of denoised images, as shown in Figure 7. First, we perform a local normalization for a gray image and then sample nonoverlapping image blocks where the size is $64 \times 64$ pixels from normalized image. Our network consists of ten layers: $64 \times 64-32 \times 30 \times 30-32 \times 15 \times 15-96 \times$ $15 \times 15-96 \times 7 \times 7-128 \times 7 \times 7-128 \times 3 \times 3-800-800-1$. We apply a dropout regularization with a ratio of 0.5 after the second fully connected layers. Finally, we obtain average scores of all of the image blocks to represent the entire image quality score.

3.4.2. Image Denoising Algorithm. The ROF model is one of the most effective methods for image denoising. It aims to model the problem of image denoising as a minimization of the energy function to make the image smooth while the edge can be maintained well. Generally, the total variation value of the noisy image is larger than the clear image. The effect of noise removal can be achieved by solving the minimization function of the total variance. Therefore, the ROF model for image denoising is as follows:

$$
\operatorname{argmin}\left\{\frac{\mu}{2}\|u-f\|_{2}^{2}+\|u\|_{T V}\right\}
$$

where $f$ is the noise image and $u$ is the denoising image. The first term of the above formula is the fidelity term, so that the denoised image $u$ keeps as much of the information in the noise image $f$ as possible. The second item is the TV regular term, which allows the model to effectively preserve edges while denoising. $\mu>0$ is a regularization parameter. The larger $\mu$ is, the smoother the denoising image will be, resulting in blurred image. The smaller $\mu$ is, the worse the denoising effect is. Therefore, choosing a suitable $\mu$ has a great effect on the results of the denoising. There are two cases of total variance. In this section, we only consider the isotropic ROF denoising problem:

$$
\min \left\{\left\|\sqrt{\left(\nabla_{x} u\right)^{2}+\left(\nabla_{y} u\right)^{2}}\right\|_{1}+\frac{\mu}{2}\|u-f\|_{2}^{2}\right\}
$$

Split Bregman method [22] is used to solve formula (9); assuming $\mathrm{d}_{\mathrm{x}} \approx \nabla_{\mathrm{x}} \mathrm{u}$ and $\mathrm{d}_{\mathrm{y}} \approx \nabla_{\mathrm{y}} \mathrm{u}$, the image denoising problem is as follows:

$$
\begin{aligned}
& \min \left\|\left(d_{x}, d_{y}\right)\right\|_{2}+\frac{\mu}{2}\|u-f\|_{2}^{2}+\frac{\lambda}{2}\left\|d_{x}-\nabla_{x} u-b_{x}\right\|_{2}^{2} \\
& +\frac{\lambda}{2}\left\|d_{y}-\nabla_{y} u-b_{y}\right\|_{2}^{2}
\end{aligned}
$$

Using Bregman iterative algorithm to solve (10), the isotropic ROF algorithm is as shown in Algorithm 1.

$G$ denotes solving the linear equations with Gauss-Seidel iteration. We focus on the influence of parameter selection on the performance of the total variation denoising algorithm.

3.4.3. The Framework of Parameter Selection. In order to study the effect of parameter selection based on image quality on denoising results, we denoise the Park image [23] with 25 different values of parameter $\mu$. The values are uniformly 


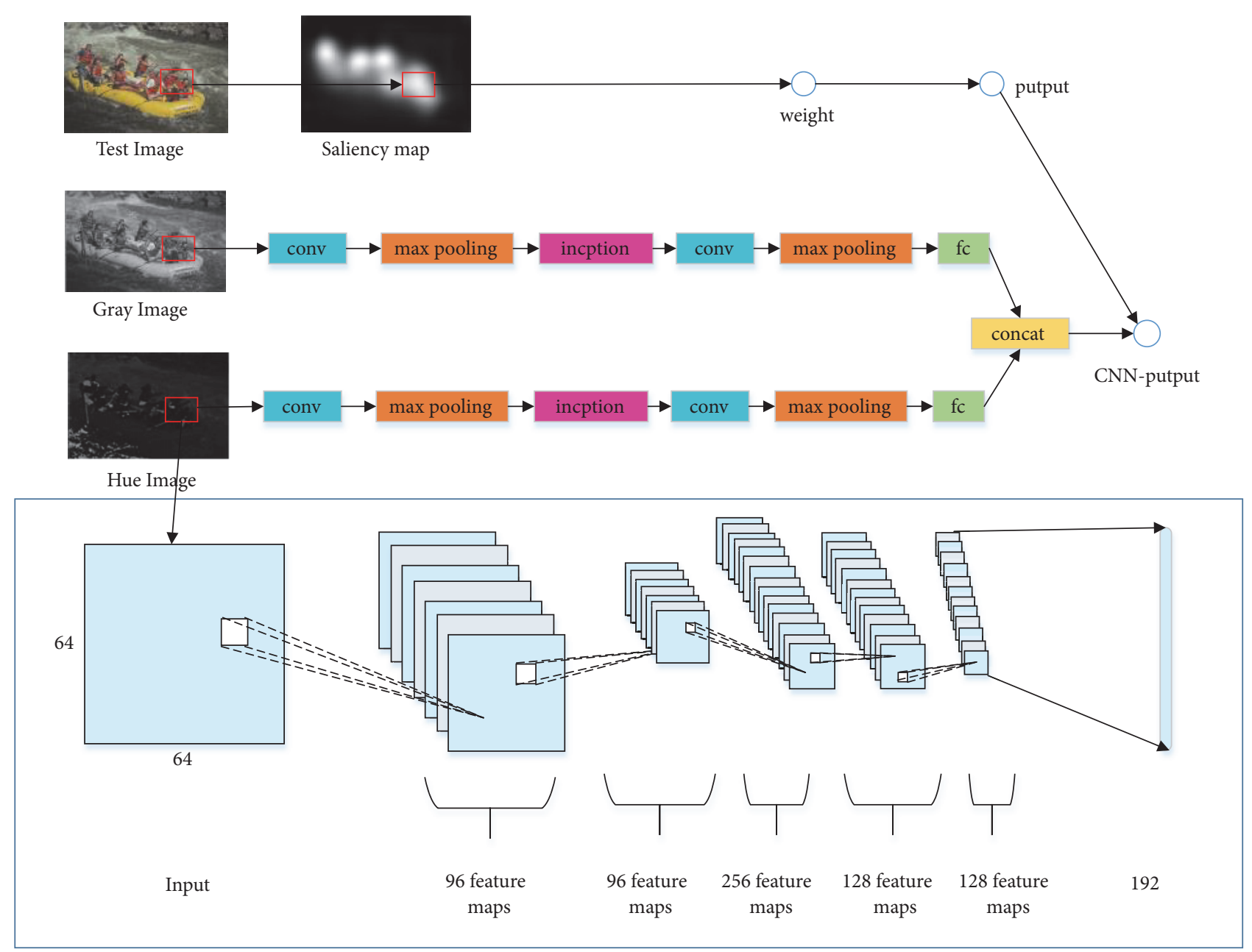

FIgURE 6: Algorithm architecture.

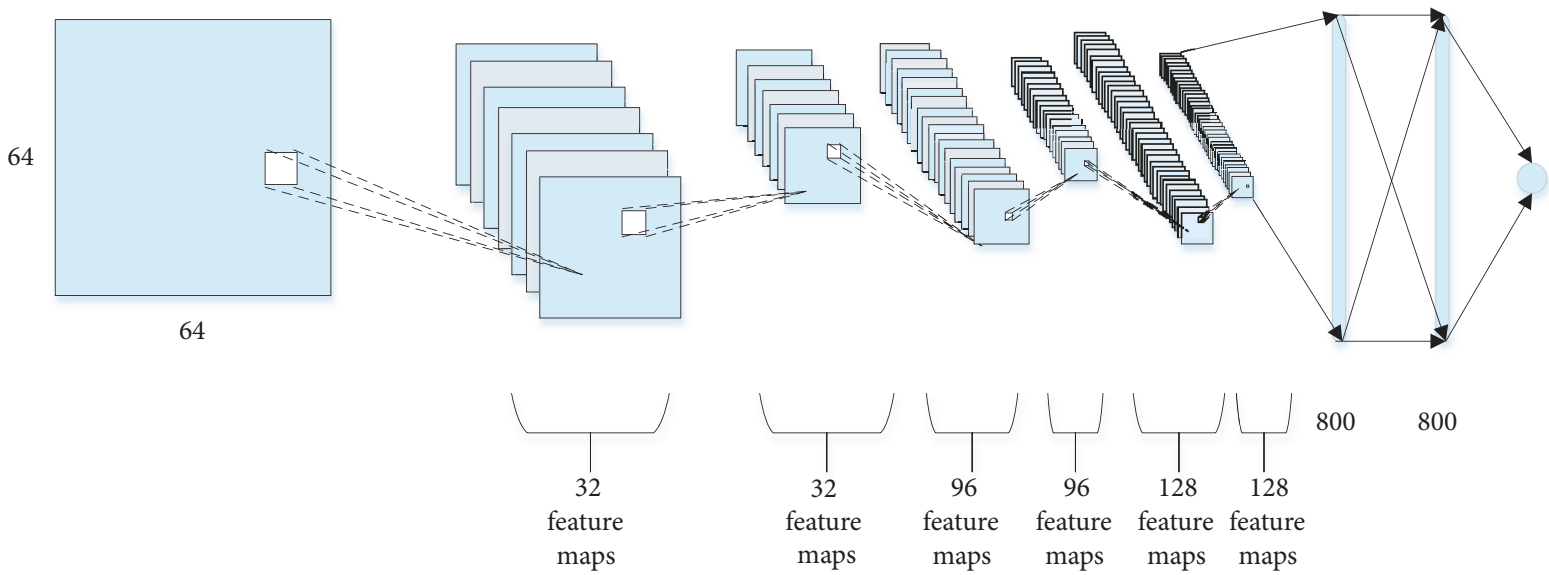

Figure 7: CNN model architecture.

sampled from 1 to 49 . Figure 8 (a) shows the variation of the denoised image quality in the iterative process for different parameter values. Figure 8 (b) is the result of the denoised image obtained using the SSIM and CNN Q methods to evaluate different parameters. Their predicted image quality trends are the same. It is obvious that the best denoising effect is obtained when parameter $\mu$ is 13 .

However, image quality assessment is performed after the completion of the whole processing of iterative convergence when the range of parameter is large. Therefore, choosing an 


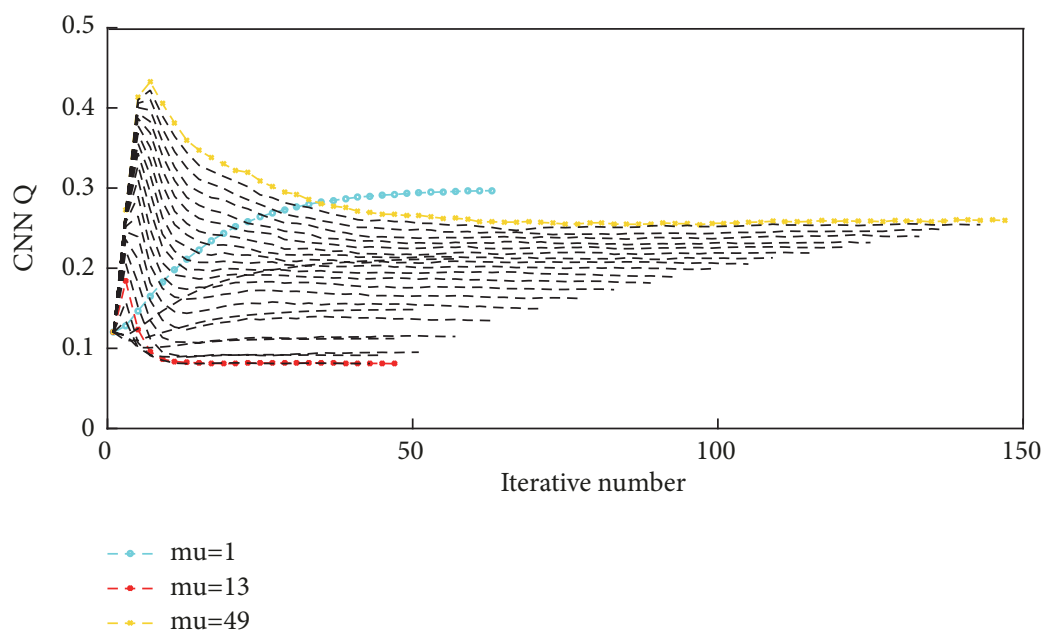

(a)

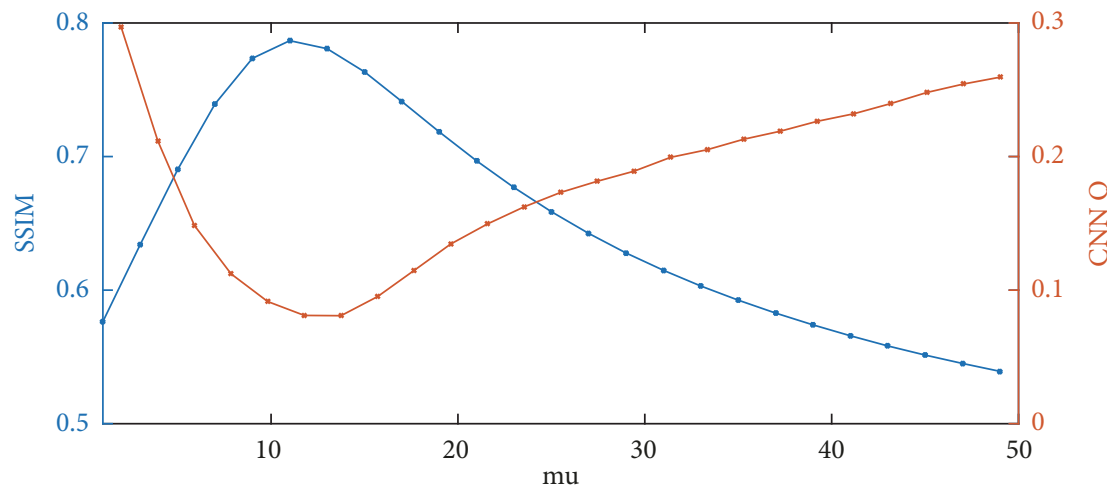

$\rightarrow$ SSIM

$\rightarrow$ CNN Q

(b)

FIGURE 8: (a) Iterative process of different parameter values; (b) using SSIM and CNN Q to evaluate the denoising image quality of different parameter values.

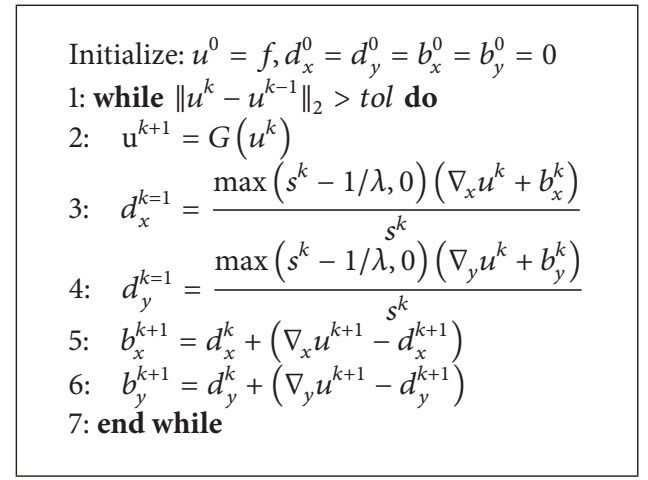

Algorithm 1

optimum parameter in a certain range requires a very large number of iterations.

Therefore, we use the changes in iteratively generated denoising image quality as a basis for determining if

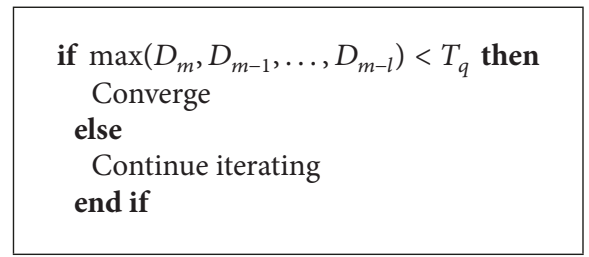

Algorithm 2

the iterative algorithm converges. Assuming that $\mathrm{Dm}=$ $\mathrm{Q}(\mathrm{m}+1)-\mathrm{Q}(\mathrm{m}), \mathrm{Q}(\mathrm{m})$ represents the quality score of the denoised image produced by the $\mathrm{m}$-th iteration, and the algorithm convergence is presented in Algorithm 2.

"l" denotes the iterative length of the change of image quality that needs to be taken into account to ensure that the algorithm converges and produces a permissible change in image quality. The larger the " $l$ " value is, the smaller the " $T q$ " value becomes, indicating that the convergence condition of the algorithm is stricter. 


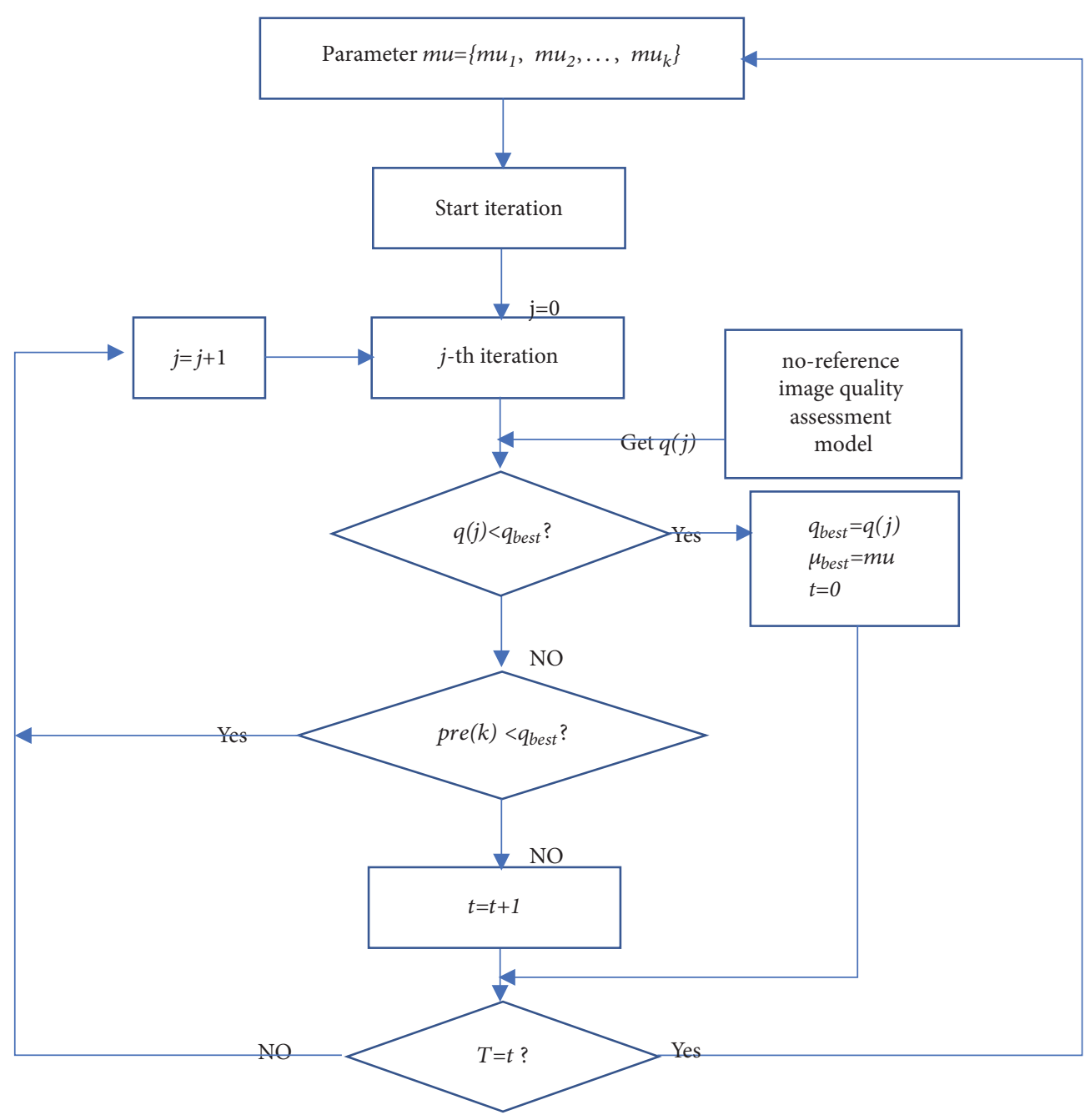

FIGURE 9: Framework of parameter selection.

Our improvement to the ROF algorithm is as follows. The algorithm can determine whether the parameter value is the optimal parameter we need before convergence, as shown in Figure 9.

Suppose pre $(k)=q(j)+\operatorname{dev}(j) * l_{\text {pre }} ; \operatorname{pre}(k)$ is the predicted quality value of the parameter $k . q(j)$ represents the quality of the denoised image from the $j$ th iteration. $\operatorname{dev}(j)$ is the numerical derivative of the $j$ th iteration. $l_{\text {pre }}$ is the predicted iteration length.

Suppose $q_{\text {best }}$ is the quality score of denoised image. And $t$ is the count and $T$ is the threshold.

At the end of each iteration of the ROF algorithm, we use the above-mentioned evaluation algorithm to evaluate the quality of the denoising image $q(j)$. In order to obtain an optimal-quality image, $q(j)$ is compared with the quality of the best image $q_{\text {best }}$ obtained previously. The iterative process terminates if the quality of the denoised image resulting from $T$ consecutive iterations is worse than $q_{b e s t}$. At this time we believe that the current parameter has achieved the best denoising effect. More iterations of the current parameter are unnecessary. However, there may be an abrupt change in the quality of the image produced by subsequent iterations, so the judgment condition pre $(k)<q_{\text {best }}$ is increased to prevent misjudgment. Next, we need to iteratively evaluate other parameters in the same way.

If we want to further improve the performance of the parameter selection framework, we can simplify the complexity of the image evaluation network model, as described in Section 3.4.1, to speed up the evaluation time at the expense of minor loss of precision. Threshold $T$ relates to the stringency of the convergence conditions. Finding a reasonable T-value for the speed and accuracy is helpful for the algorithm. In addition, we can also reduce the number of candidate parameters by reducing the fineness of the range division of the parameter $\mu$ within an acceptable range.

\section{Results and Analysis}

4.1. Image Quality Assessment Experiment. We obtain the quality evaluation of the image by weighting the quality score of the sampled image block. Therefore, the sampling strategy can affect the overall algorithm performance. In this section, 
TABLE 1: LCC and SROCC with different patch size.

\begin{tabular}{lccccc}
\hline Size & 80 & 72 & 64 & 56 & 48 \\
LCC & 0.967 & 0.966 & 0.964 & 0.958 & 0.953 \\
SROCC & 0.975 & 0.964 & 0.962 & 0.957 & 0.951 \\
\hline
\end{tabular}

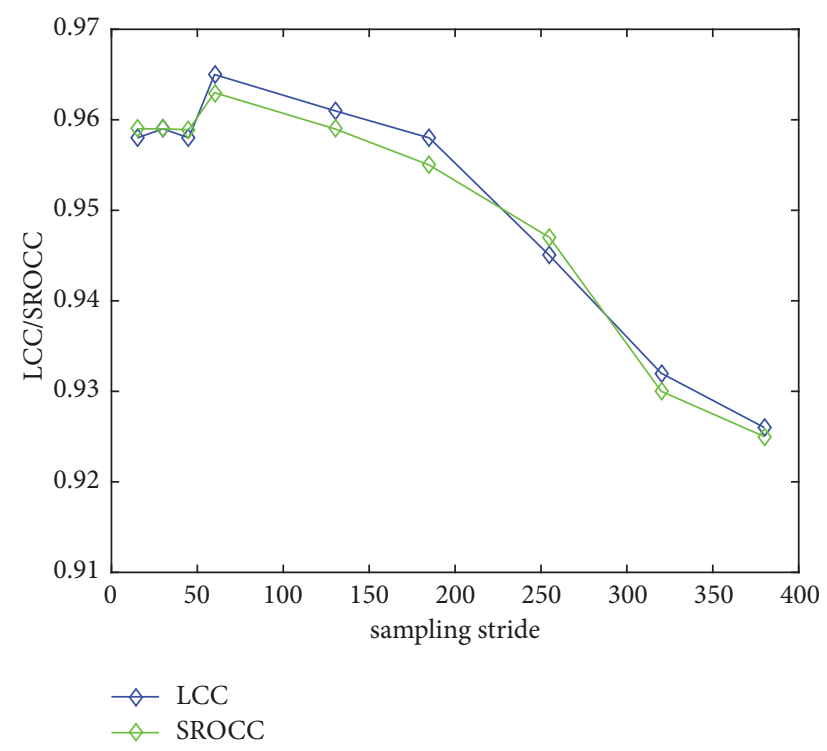

FIGURE 10: LCC and SROCC with different sampling stride.

we study the effect of two parameters on the performance of the algorithm: the block size of the sampled image and the number of samples per image.

(1) Block size: we assume the sampled image is nonoverlapped. In order to study the effect of image block size on the performance of our algorithm, we use fixed sampling strides and different image block sizes. The results are shown in Table 1 . We find that the performance increases slightly with the increasement of patch size. Moreover, the performance of the algorithm will begin to decrease if the size is very large because the local information of the image has been lost. Here, we choose the size of $64 \times 64$.

(2) Sampling stride: we study the effect of the number of image blocks sampled on each image. We use a fixed block size of $64 \times 64$ and various strides. Figure 10 shows that different sampling strides lead to changes in the performance of the algorithm. We have found that the number of image blocks seriously affects the performance of the algorithm. In general, a larger stride leads to lower performance because less image information is used to evaluate image quality.

In order to test the performance of our proposed algorithm, we conducted experiments on the LIVE database [24]. In order to ensure the robustness of our proposed method, we performed 100 training and testing experiments, independently of the image content and the specific training test set. For each experiment, we randomly selected distorted images corresponding to $60 \%$ of the reference images in the LIVE database as the training set, $20 \%$ distorted image as the verification set, and the remaining images as the test set.
Therefore, all the results shown in this chapter are averages of the results of these 100 experiments.

Tables 2 and 3 are the results of comparing our algorithm to the existing representative image quality assessment methods, including no-reference image quality evaluation methods and reference image quality evaluation methods. No-reference methods include DIIVINE [24], BLIINDS-II [25], BRISQUE, CORNIA [26], and CNN.

It can be seen that the method we propose has a high degree of consistency with human subjective evaluation. Compared to other no-reference image quality assessment methods, our method has the best performance on the three distortion types, JP2K, WN, and FF.

In order to test the generalization ability of our proposed algorithm, we conducted an experiment on the TID2008 database. We train our model on the entire LIVE database and then test model on the TID2008 database. In Table 4, the LCC indicator shows that our algorithm's performance is slightly better than those of other algorithms, similarly to the CNN method in [16]. The SROCC value of our algorithm is very high, which is obviously better than the performance of the other algorithms. In general, the independence of our proposed algorithm is strong.

4.2. Algorithm Evaluation Experiment. First, we evaluate the performance of our proposed evaluation algorithm. We train our CNN model on the LIVE database and test the performance of model on the TID2008 database. Our training data is $\mathrm{WN}$ and blur types of distortion images from the LIVE database. By default, all results reported are averaged from 100 train-test iterations. In each iteration, we randomly select $80 \%$ distorted images as the training set and the remaining $20 \%$ as the validation set. The result is shown as Table 5 .

We apply the parameter selection framework to the ROF denoising model. First, we use different parameters to denoise the two images and observe the denoising results. Figure 11 presents the denoising results of the Park image at three different parameter values. For Park images, the image denoising effect is better than that of other values when the $\mu$ parameter value is 13 .

Next, we study the influence of the parameter selection framework on the iterative denoising algorithm. In our experiment, we set $T_{q}=0.001, l=5, l_{\text {pre }}=5$, and $T=10$. We evaluate the quality of the denoised image produced by each iteration to predict the quality of the denoised image during subsequent iterations. Figure 11 is the result of comparative experiments with a parameter selection framework and with no parameter selection framework for denoising a Park graph. As shown in Figure 12, with a denoising algorithm that uses a parameter selection framework, the number of iterations per parameter is significantly reduced. Moreover, the optimal parameter values selected by the two experiments are the same, which indicates that our proposed parameter selection framework not only greatly reduces the number of iterations of the algorithm, but also accurately selects the optimal parameter value.

In order to further prove this conclusion, we calculated the data generated by the denoising experiments on multiple 
TABLE 2: Median SROCC across 100 train-test iterations on the LIVE database.

\begin{tabular}{lccccrr}
\hline SROCC & JP2K & JPEG & WN & BLUR & FF & 0.874 \\
\hline PSNR & 0.870 & 0.885 & 0.942 & 0.763 & 0.907 & 0.941 \\
SSIM & 0.939 & 0.946 & 0.964 & 0.967 & 0.972 & 0.949 \\
FSIM & 0.970 & 0.981 & 0.964 \\
\hline DIIVINE & 0.913 & 0.910 & 0.984 & 0.921 & 0.863 & 0.916 \\
BLIINDS-II & 0.929 & 0.942 & 0.969 & 0.923 & 0.889 & 0.931 \\
BRISQUE & 0.914 & 0.965 & 0.979 & 0.951 & 0.877 & 0.940 \\
CORAIN & 0.943 & 0.955 & 0.976 & 0.969 & 0.906 & 0.942 \\
CNN & 0.952 & $\mathbf{0 . 9 7 7}$ & 0.978 & 0.962 & 0.956 \\
Proposed & $\mathbf{0 . 9 6 7}$ & 0.968 & $\mathbf{0 . 9 8 6}$ & & $\mathbf{0 . 9 3 1}$ \\
\hline
\end{tabular}

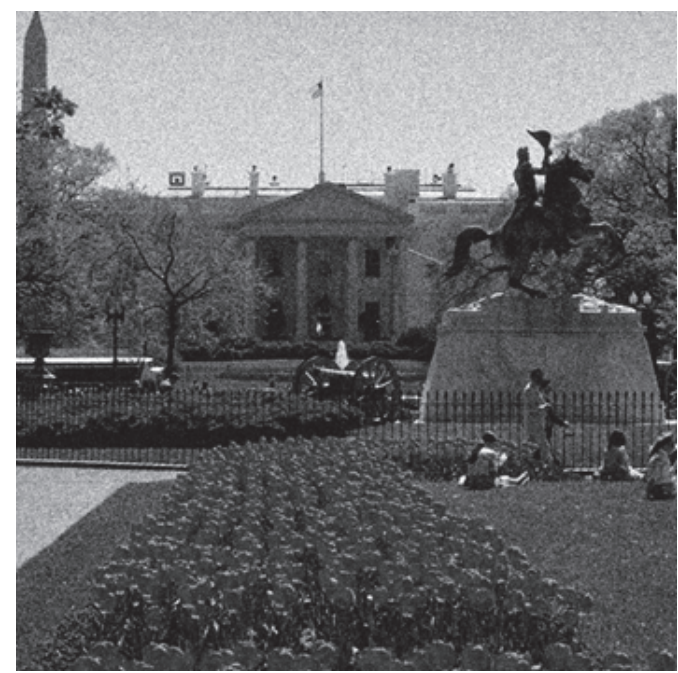

(a)

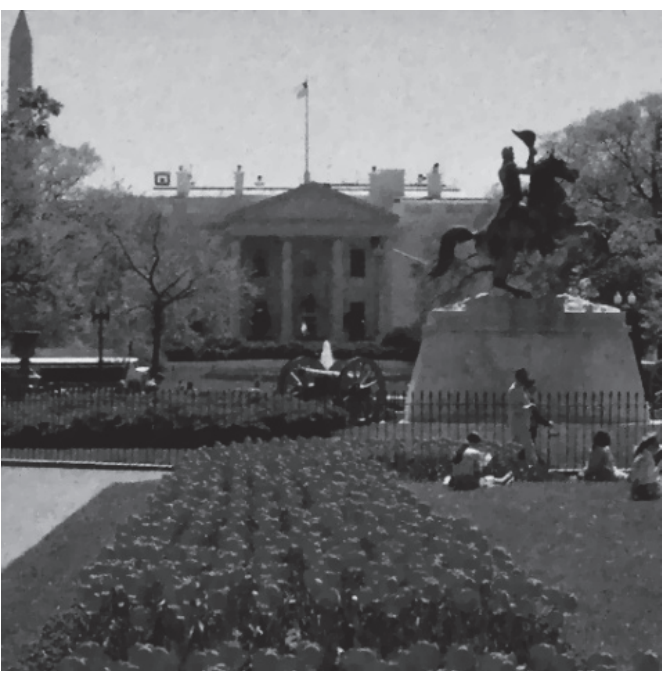

(c)

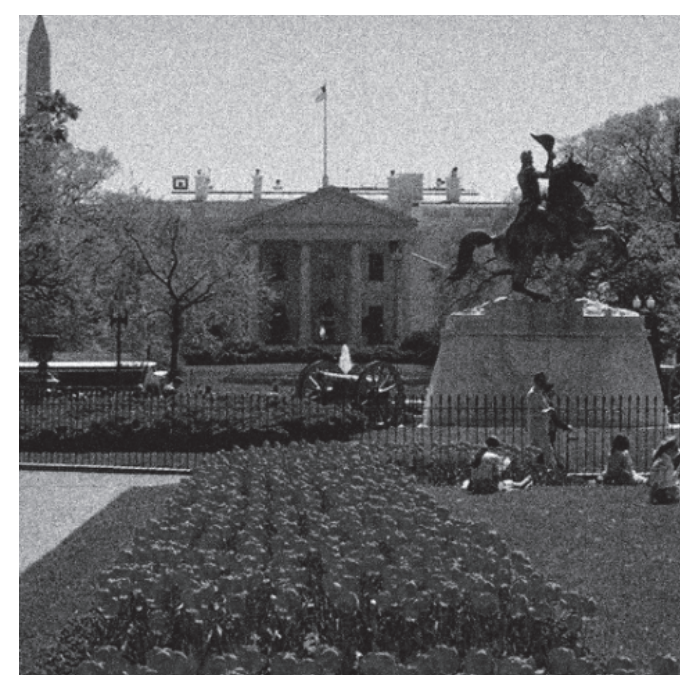

(b)

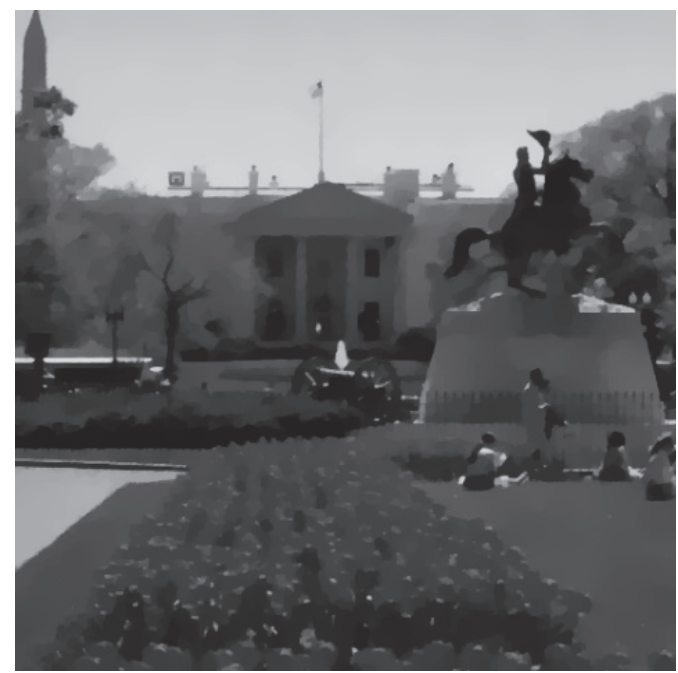

(d)

FIGURE 11: (a) Park image of noise image; (b) denoising result with $\mu$ parameter value equal to 1; denoising result with $\mu$ parameter equal to 13; (d) denoising result with $\mu$ parameter equal to 49 . 


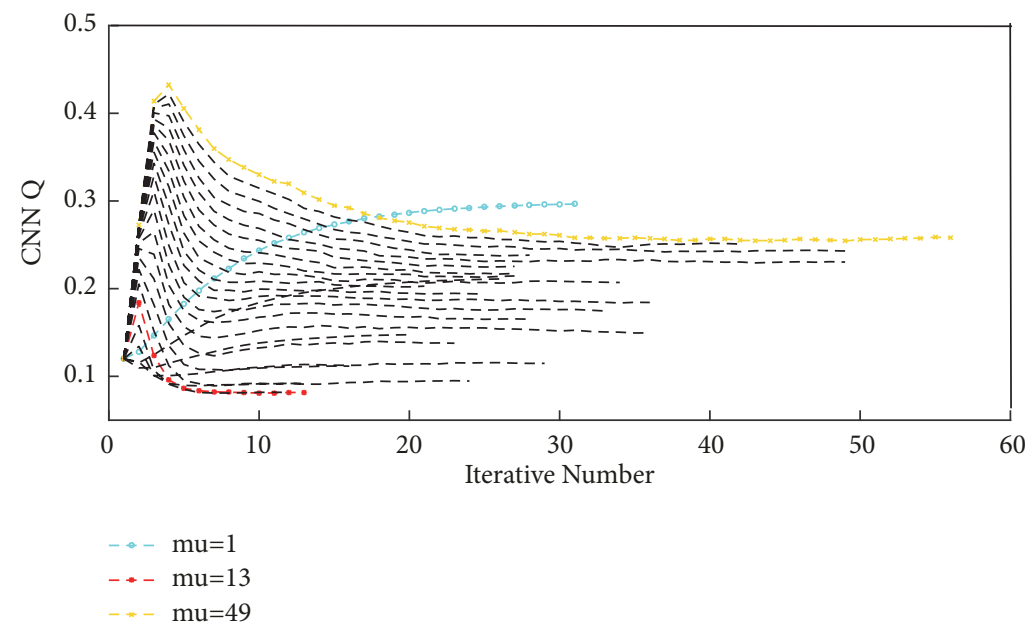

(a)

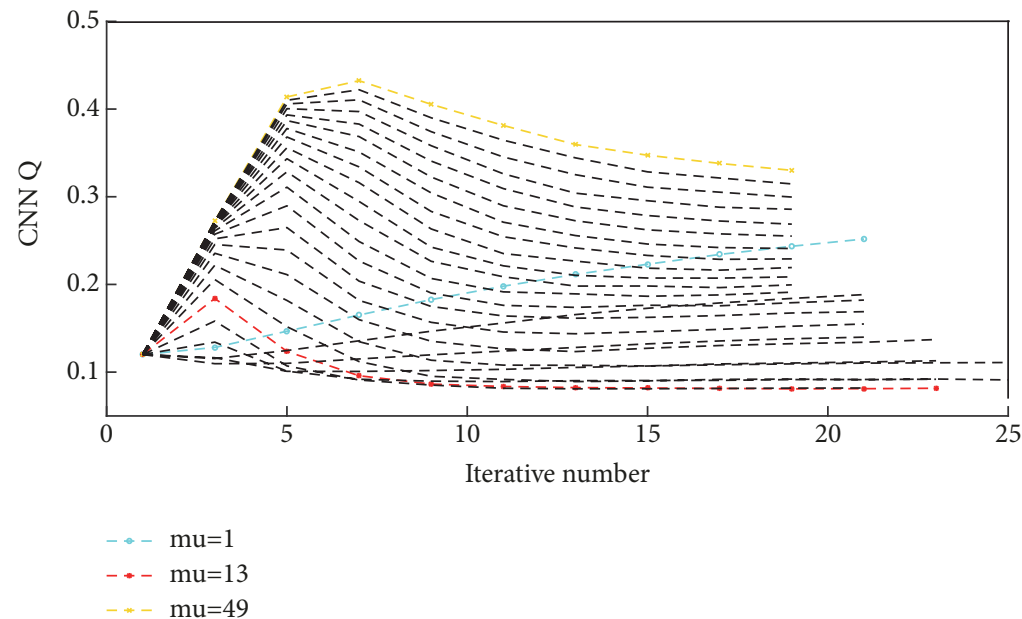

(b)

FIGURE 12: Park diagram, (a) ROF denoising process without parameter selection framework; (b) ROF denoising process with parameter selection framework.

TABLE 3: Median LCC across 100 train-test iterations on the LIVE database.

\begin{tabular}{|c|c|c|c|c|c|c|}
\hline LCC & JP2K & JPEG & $\mathrm{WN}$ & BLUR & $\mathrm{FF}$ & ALL \\
\hline $\begin{array}{l}\text { PSNR } \\
\end{array}$ & 0.873 & 0.876 & 0.926 & 0.779 & 0.870 & 0.856 \\
\hline SSIM & 0.921 & 0.955 & 0.982 & 0.893 & 0.939 & 0.906 \\
\hline FSIM & 0.910 & 0.985 & 0.976 & 0.978 & 0.912 & 0.960 \\
\hline DIIVINE & 0.922 & 0.921 & 0.988 & 0.923 & 0.888 & 0.917 \\
\hline BLIINDS-II & 0.935 & 0.968 & 0.980 & 0.938 & 0.896 & 0.930 \\
\hline BRISQUE & 0.922 & 0.973 & 0.985 & 0.951 & 0.903 & 0.942 \\
\hline CORAIN & 0.951 & 0.965 & 0.987 & 0.968 & 0.917 & 0.935 \\
\hline $\mathrm{CNN}$ & 0.953 & 0.981 & 0.984 & 0.953 & 0.933 & 0.953 \\
\hline Proposed & 0.974 & 0.975 & 0.991 & 0.963 & 0.953 & 0.964 \\
\hline
\end{tabular}

TABLE 4: Experimental LCC and SROCC indicators on the TID2008 database.

\begin{tabular}{lcccc}
\hline & BRISQUE & CORNIA & CNN & Proposed \\
\hline LCC & 0.882 & 0.892 & 0.920 & $\mathbf{0 . 9 2 2}$ \\
SROCC & 0.892 & 0.880 & 0.903 & $\mathbf{0 . 9 2 1}$ \\
\hline
\end{tabular}


TABLE 5: Results on LIVE validation sets and TID2008 test sets.

\begin{tabular}{lcc}
\hline & LCC & SROCC \\
\hline LIVE validation set & 0.966 & 0.957 \\
TID2008 testing set & 0.9161 & 0.9013 \\
\hline
\end{tabular}

TABLE 6: Performance of our parameter selection framework.

\begin{tabular}{|c|c|c|c|c|c|c|c|}
\hline & \multicolumn{3}{|c|}{ Parameter selection framework } & \multicolumn{3}{|c|}{ without parameter selection framework } & \multirow{2}{*}{ Reduced iteration } \\
\hline & $\mu$ best of $\mathrm{A}$ & Iterations of A & CPU time of $\mathrm{A}(\mathrm{s})$ & $\mu$ best of $\mathrm{B}$ & Iterations of B & CPU time of B(s) & \\
\hline Park & 13 & 259 & 40.125 & 13 & 658 & 79.528 & $60.7 \%$ \\
\hline Fisher & 11 & 238 & 37.524 & 11 & 932 & 122.45 & $74.5 \%$ \\
\hline Pepper & 21 & 245 & 37.283 & 21 & 709 & 88.194 & $65.4 \%$ \\
\hline Man & 11 & 217 & 30.478 & 11 & 707 & 86.526 & $69.3 \%$ \\
\hline Cameraman & 13 & 274 & 45.589 & 13 & 1063 & 132.36 & $74.2 \%$ \\
\hline Barbara & 13 & 235 & 34.235 & 13 & 817 & 106.75 & $71.2 \%$ \\
\hline
\end{tabular}

images. The experimental results are shown in Table 6. Obviously, they are consistent with the above findings. According to experiments, the running time of the improved ROF algorithm is much lower than that of the original algorithm. In other words, the computational cost of the algorithm mainly comes from the iteration; i.e., the computational cost is proportional to the number of iterations. We improve the algorithm performance by reducing the number of iterations while ensuring the optimal parameters are obtained

\section{Conclusions}

We propose a cascaded algorithm for no-reference image quality assessment and image denoising based on $\mathrm{CNN}$ and visual saliency, to be applied in image security and authorization. This algorithm uses a strategy of significantly weighting image blocks to obtain a quality assessment more in line with the human visual system. This method has achieved very good performance on the LIVE database. The evaluation method is embedded into an image denoising algorithm, such as the total variation denoising algorithm. We propose a parameter selection framework that can judge the advantages and disadvantages of the parameters according to the quality change of the denoising image generated during the iterative process. And it is verified that the iteration number of denoising process is significantly reduced by parameter selection. Meanwhile, we can accurately find the best parameters from a bunch of parameter candidates.

\section{Data Availability}

The data used to support the findings of this study are included within the article.

\section{Conflicts of Interest}

The authors declare that they have no conflicts of interest.

\section{References}

[1] Z. Huang, S. Liu, X. Mao, K. Chen, and J. Li, "Insight of the protection for data security under selective opening attacks," Information Sciences, vol. 412-413, pp. 223-241, 2017.

[2] L. Sun, Z. Li, Q. Yan, W. Srisa-an, and Y. Pan, "SigPID: significant permission identification for android malware detection," in Proceedings of the 2016 11th International Conference on Malicious and Unwanted Software (MALWARE), pp. 1-8, Fajardo, PR, USA, October 2016.

[3] Y. Zhang, X. Chen, J. Li, D. S. Wong, H. Li, and I. You, "Ensuring attribute privacy protection and fast decryption for outsourced data security in mobile cloud computing," Information Sciences, vol. 379, pp. 42-61, 2017.

[4] C. Yan, H. Xie, S. Liu, J. Yin, Y. Zhang, and Q. Dai, "Effective Uyghur Language Text Detection in Complex Background Images for Traffic Prompt Identification," IEEE Transactions on Intelligent Transportation Systems, vol. 19, no. 1, pp. 220-229, 2018.

[5] Q. Xu, T. Jiang, Y. Yao, Q. Huang, B. Yan, and W. Lin, "Random partial paired comparison for subjective video quality assessment via hodgerank," in Proceedings of the 19th ACM International Conference on Multimedia ACM Multimedia 2011, MM'11, pp. 393-402, USA, December 2011.

[6] L. L. Thurstone, "A law of comparative judgment," Psychological Review, vol. 34, no. 4, pp. 273-286, 1927.

[7] K. Chen, C. Wu, Y. Chang, and C. Lei, "A crowdsourceable QoE evaluation framework for multimedia content," in Proceedings of the the seventeen ACM international conference, p. 491, Beijing, China, October 2009.

[8] W.-S. Lai, J.-B. Huang, Z. Hu, N. Ahuja, and M.-H. Yang, "Comparative study for single image blind deblurring," in Proceedings of the 2016 IEEE Conference on Computer Vision and Pattern Recognition, CVPR 2016, pp. 1701-1709, USA, July 2016.

[9] H. Liang and D. S. Weller, "Comparison-based image quality assessment for selecting image restoration parameters," IEEE Transactions on Image Processing, vol. 25, no. 11, pp. 5118-5130, 2016.

[10] X. Chen, S. Chen, and Y. Wu, "Coverless information hiding method based on the Chinese character encoding," Journal of Internet Technology, vol. 18, no. 2, pp. 91-98, 2017. 
[11] J. L. Herraiz, S. Gabarda, and G. Cristobal, "Automatic parameter selection in PET image reconstruction based on noreference image quality assessment," in Proceedings of the 2012 IEEE Nuclear Science Symposium and Medical Imaging Conference Record, NSS/MIC 2012, pp. 3371-3374, USA, November 2012.

[12] X. Zhu and P. Milanfar, "Automatic parameter selection for denoising algorithms using a no-reference measure of image content," IEEE Transactions on Image Processing, vol. 19, no. 12, pp. 3116-3132, 2010.

[13] H. Liang and D. S. Weller, "Regularization parameter trimming for iterative image reconstruction," in Proceedings of the 49th Asilomar Conference on Signals, Systems and Computers, ACSSC 2015, pp. 755-759, USA, November 2015.

[14] J. Li, L. Xu, H. Li, C. Chang, and F. Sun, "Parameter Selection for Denoising Algorithms Using NR-IQA with CNN," in MultiMedia Modeling, vol. 10704 of Lecture Notes in Computer Science, pp. 381-392, Springer International Publishing, Cham, 2018.

[15] A. Mittal, A. K. Moorthy, and A. . Bovik, "No-reference image quality assessment in the spatial domain," IEEE Transactions on Image Processing, vol. 21, no. 12, pp. 4695-4708, 2012.

[16] L. Kang, P. Ye, Y. Li, and D. Doermann, "Convolutional neural networks for no-reference image quality assessment," in Proceedings of the 27th IEEE Conference on Computer Vision and Pattern Recognition, CVPR 2014, pp. 1733-1740, USA, June 2014.

[17] D. L. Ruderman, "The statistics of natural images," Networks, vol. 5, pp. 517-548, 1994.

[18] S. Bosse, D. Maniry, T. Wiegand, and W. Samek, "A deep neural network for image quality assessment," in Proceedings of the 23rd IEEE International Conference on Image Processing, ICIP 2016, pp. 3773-3777, USA, September 2016.

[19] A. Buades, B. Coll, and J. M. Morel, "A review of image denoising algorithms, with a new one," Multiscale Modeling and Simulation: A SIAM Interdisciplinary Journal, vol. 4, no. 2, pp. 490-530, 2005.

[20] K. Dabov, A. Foi, V. Katkovnik, and K. Egiazarian, "Image denoising by sparse 3-D transform-domain collaborative filtering," IEEE Transactions on Image Processing, vol. 16, no. 8, pp. 2080-2095, 2007.

[21] L. I. Rudin, S. Osher, and E. Fatemi, "Nonlinear total variation based noise removal algorithms," Physica D: Nonlinear Phenomena, vol. 60, no. 1-4, pp. 259-268, 1992.

[22] T. Goldstein and S. Osher, "The Split Bregman Method for L1Regularized Problems," SIAM Journal on Imaging Sciences, vol. 2, no. 2, pp. 323-343, 2009.

[23] E. C. Larson and D. M. Chandler, "Most apparent distortion: full-reference image quality assessment and the role of strategy," Journal of Electronic Imaging, vol. 19, no. 1, Article ID 011006, 2010.

[24] H. Sheikh, A. Bovik, and L. Cormack, "No-reference quality assessment using natural scene statistics: JPEG2000," IEEE Transactions on Image Processing, vol. 14, no. 11, pp. 1918-1927, 2005.

[25] M. A. Saad, A. C. Bovik, and C. Charrier, "Blind image quality assessment: a natural scene statistics approach in the DCT domain," IEEE Transactions on Image Processing, vol. 21, no. 8, pp. 3339-3352, 2012.

[26] P. Ye, J. Kumar, L. Kang, and D. Doermann, "Unsupervised feature learning framework for no-reference image quality assessment," in Proceedings of the 2012 IEEE Conference on Computer Vision and Pattern Recognition, CVPR 2012, pp. 10981105, USA, June 2012. 


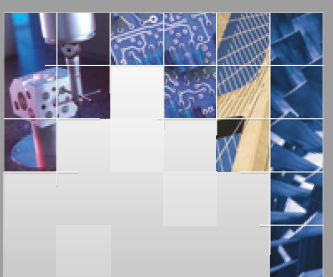

\section{Enfincering}
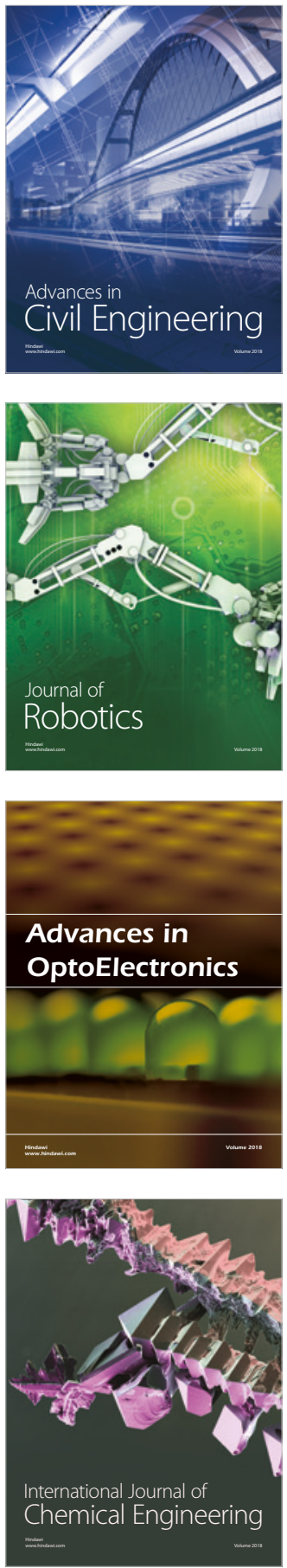

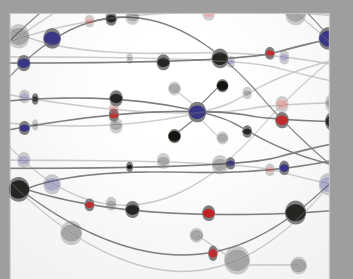

\section{Rotating \\ Machinery}

The Scientific World Journal

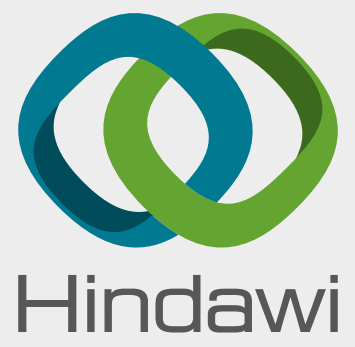

Submit your manuscripts at

www.hindawi.com
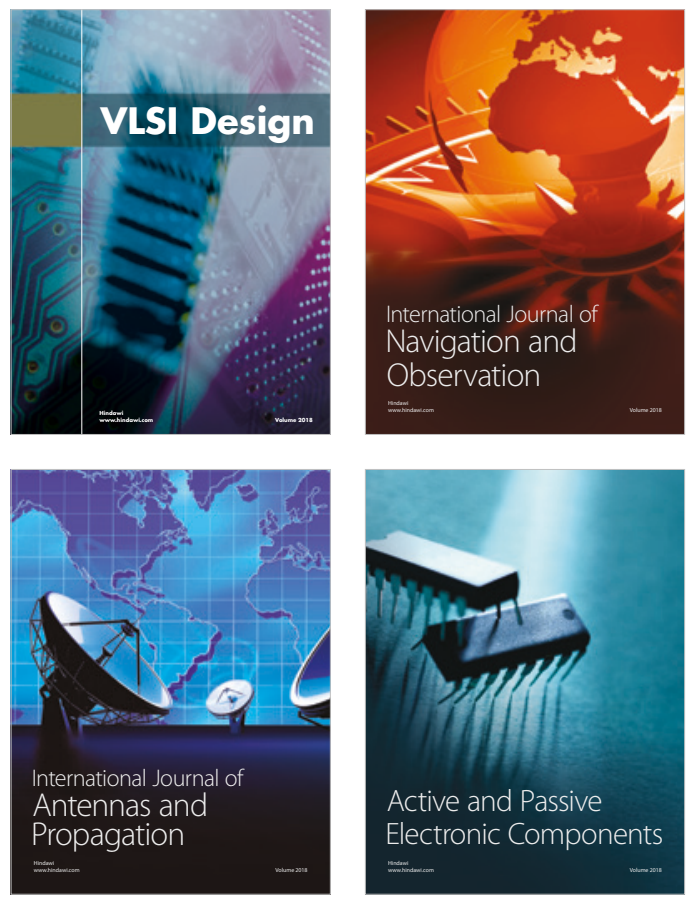
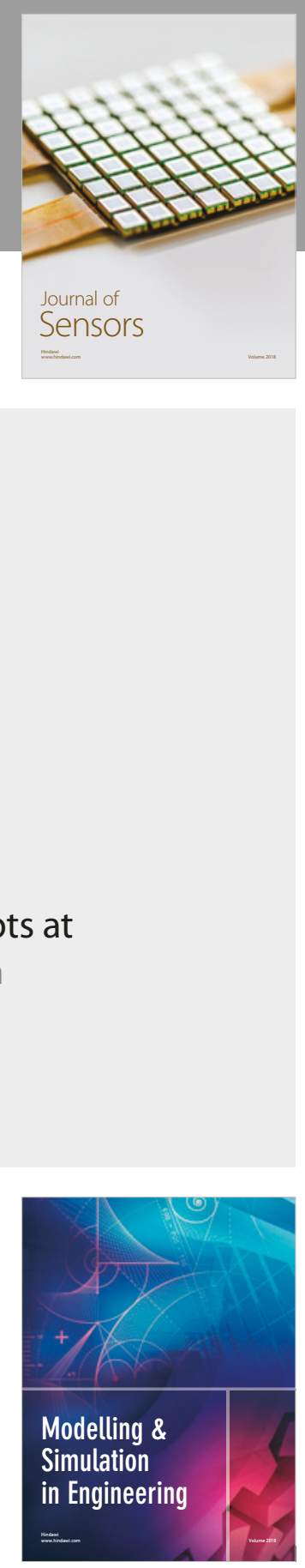

\section{Advances \\ Multimedia}
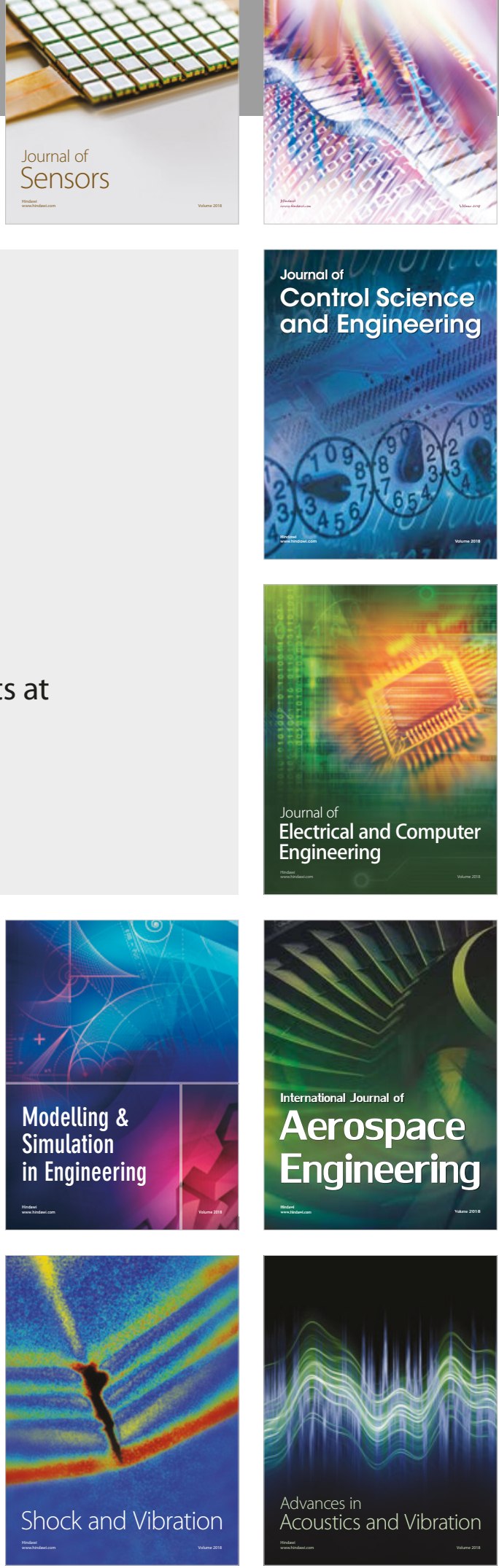